\title{
On the twisted Daehee polynomials with $q$-parameter
}

\section{Jin-Woo Park}

"Correspondence: a0417001@knu.ac.kr Department of Mathematics Education, Sehan University, YoungAm-gun, Chunnam, 526-702, Republic of Korea

\begin{abstract}
The $n$th twisted Daehee numbers with $q$-parameter are closely related to higher-order Bernoulli numbers and Bernoulli numbers of the second kind. In this paper, we give a $p$-adic integral representation of the twisted Daehee polynomials with $q$-parameter, and we derive some interesting properties related to the $n$th twisted Daehee polynomials with q-parameter.
\end{abstract}

Keywords: Bernoulli polynomials; Daehee polynomials with $q$-parameter; $p$-adic invariant integral

\section{Introduction}

Let $p$ be a fixed prime number. Throughout this paper, $\mathbb{Z}_{p}, \mathbb{Q}_{p}$, and $\mathbb{C}_{p}$ will, respectively, denote the ring of $p$-adic rational integers, the field of $p$-adic rational numbers, and the completions of algebraic closure of $\mathbb{Q}_{p}$. The $p$-adic norm is defined $|p|_{p}=\frac{1}{p}$.

When one talks of $q$-extension, $q$ is variously considered as an indeterminate, a complex $q \in \mathbb{C}$, or a $p$-adic number $q \in \mathbb{C}_{p}$. If $q \in \mathbb{C}$, one normally assumes that $|q|<1$. If $q \in \mathbb{C}_{p}$, then we assume that $|q-1|_{p}<p^{-\frac{1}{p-1}}$ so that $q^{x}=\exp (x \log q)$ for each $x \in \mathbb{Z}_{p}$. Throughout this paper, we use the notation

$$
[x]_{q}=\frac{1-q^{x}}{1-q} .
$$

Note that $\lim _{q \rightarrow 1}[x]_{q}=x$ for each $x \in \mathbb{Z}_{p}$.

Let $U D\left(\mathbb{Z}_{p}\right)$ be the space of uniformly differentiable functions on $\mathbb{Z}_{p}$. For $f \in U D\left(\mathbb{Z}_{p}\right)$, the $p$-adic invariant integral on $\mathbb{Z}_{p}$ is defined by Kim as follows:

$$
I(f)=\int_{\mathbb{Z}_{p}} f(x) d \mu_{0}(x)=\lim _{n \rightarrow \infty} \frac{1}{p^{n}} \sum_{x=0}^{p^{n}-1} f(x) \quad \text { (see [1-3]). }
$$

Let $f_{1}$ be the translation of $f$ with $f_{1}(x)=f(x+1)$. Then, by (1.1), we get

$$
I\left(f_{1}\right)=I(f)+f^{\prime}(0), \quad \text { where } f^{\prime}(0)=\left.\frac{d f(x)}{d x}\right|_{x=0} .
$$

O2014 Park; licensee Springer. This is an Open Access article distributed under the terms of the Creative Commons Attribution License (http://creativecommons.org/licenses/by/2.0), which permits unrestricted use, distribution, and reproduction in any medium, provided the original work is properly cited. 
As is well known, the Stirling number of the first kind is defined by

$$
(x)_{n}=x(x-1) \cdots(x-n+1)=\sum_{l=0}^{n} S_{1}(n, l) x^{l}
$$

and the Stirling number of the second kind is given by the generating function to be

$$
\left(e^{t}-1\right)^{m}=m ! \sum_{l=m}^{\infty} S_{2}(l, m) \frac{t^{l}}{l !}
$$

(see [4-6]).

Unsigned Stirling numbers of the first kind are given by

$$
x^{\underline{n}}=x(x+1) \cdots(x+n-1)=\sum_{l=0}^{n}\left|S_{1}(n, l)\right| x^{l} .
$$

Note that if we replace $x$ to $-x$ in (1.3), then

$$
\begin{aligned}
(-x)_{n} & =(-1)^{n} x^{\underline{n}}=\sum_{l=0}^{n} S_{1}(n, l)(-1)^{l} x^{l} \\
& =(-1)^{n} \sum_{l=0}^{n}\left|S_{1}(n, l)\right| x^{l} .
\end{aligned}
$$

Hence $S_{1}(n, l)=\left|S_{1}(n, l)\right|(-1)^{n-l}$.

For $r \in \mathbb{N}$, the Bernoulli polynomials of order $r$ are defined by the generating function to be

$$
\left(\frac{t}{e^{t}-1}\right)^{r} e^{x t}=\sum_{n=0}^{\infty} B_{n}^{(r)}(x) \frac{t^{n}}{n !} \quad(\text { see }[1,4,7-18]) .
$$

When $x=0, B_{n}^{(r)}=B_{n}^{(r)}(0)$ are called the Bernoulli numbers of order $r$, and in the special case, $r=1, B_{n}^{(1)}(x)=B_{n}(x)$ are called the ordinary Bernoulli polynomials.

For $n \in \mathbb{N}$, let $T_{p}$ be the $p$-adic locally constant space defined by

$$
T_{p}=\bigcup_{n \geq 1} C_{p^{n}}=\lim _{n \rightarrow \infty} C_{p^{n}}
$$

where $C_{p^{n}}=\left\{\omega \mid \omega^{p^{n}}=1\right\}$ is the cyclic group of order $p^{n}$.

We assume that $q$ is an indeterminate in $\mathbb{C}_{p}$ with $|1-q|_{p}<p^{-\frac{1}{p-1}}$. Then we define the $q$-analog of a falling factorial sequence as follows:

$$
(x)_{n, q}=x(x-q)(x-2 q) \cdots(x-(n-1) q) \quad(n \geq 1), \quad(x)_{0, q}=1 .
$$

Note that

$$
\lim _{q \rightarrow 1}(x)_{n, q}=(x)_{n}=\sum_{l=0}^{n} S_{1}(n, l) x^{l}
$$


Recently, DS Kim and T Kim introduced the Daehee polynomials as follows:

$$
D_{n}(x)=\int_{\mathbb{Z}_{p}}(x+y)_{n} d \mu_{0}(y) \quad(n \geq 0)(\text { see }[2,9,19]) .
$$

When $x=0, D_{n}=D_{n}(0)$ are called the $n$th Daehee numbers. From (1.8), we can derive the generating function to be

$$
\left(\frac{\log (1+t)}{t}\right)(1+t)^{x}=\sum_{n=0}^{\infty} D_{n}(x) \frac{t^{n}}{n !} \quad(\text { see [9] })
$$

In addition, DS Kim et al. consider the Daehee polynomials with q-parameter, which are defined by the generating function to be

$$
\sum_{n=0}^{\infty} D_{n, q} \frac{t^{n}}{n !}=(1+q t)^{\frac{x}{q}} \frac{\log (1+q t)}{q\left((1+q t)^{\frac{1}{q}}-1\right)} \quad(\text { see }[20,21]) .
$$

When $x=0, D_{n, q}=D_{n, q}(0)$ are called the Daehee numbers with q-parameter.

From the viewpoint of a generalization of the Daehee polynomials with $q$-parameter, we consider the twisted Daehee polynomials with q-parameter, defined to be

$$
\sum_{n=0}^{\infty} D_{n, \xi, q} \frac{t^{n}}{n !}=(1+q \xi t)^{\frac{x}{q}} \frac{\log (1+q \xi t)}{q\left((1+q \xi t)^{\frac{1}{q}}-1\right)},
$$

where $t, q \in \mathbb{C}_{p}$ with $|t|_{p}<|q|_{p} p^{-\frac{1}{p-1}}$ and $\xi \in T_{p}$.

In this paper, we give a $p$-adic integral representation of the twisted Daehee polynomials with $q$-parameter, which is called the Witt-type formula for the twisted Daehee polynomials with $q$-parameter. We can derive some interesting properties related to the $n$th twisted Daehee polynomials with $q$-parameter.

\section{Witt-type formula for the $n$th twisted Daehee polynomials with q-parameter}

First, we consider the following integral representation associated with falling factorial sequences:

$$
\xi^{n} \int_{\mathbb{Z}_{p}}(x+y)_{n, q} d \mu_{0}(y), \quad \text { where } n \in \mathbb{Z}_{+}=\mathbb{N} \cup\{0\} \text { and } \xi \in T_{p} \text {. }
$$

By (2.1),

$$
\begin{aligned}
\sum_{n=0}^{\infty} \xi^{n} \int_{\mathbb{Z}_{p}}(x+y)_{n, q} d \mu_{0}(y) \frac{t^{n}}{n !} & =\sum_{n=0}^{\infty} \xi^{n} q^{n} \int_{\mathbb{Z}_{p}}\left(\frac{x+y}{q}\right)_{n} d \mu_{0}(y) \frac{t^{n}}{n !} \\
& =\int_{\mathbb{Z}_{p}}(1+q \xi t)^{\frac{x+y}{q}} d \mu_{0}(y),
\end{aligned}
$$


where $t, q \in \mathbb{C}_{p}$ with $|t|_{p}<|q|_{p} p^{-\frac{1}{p-1}}$. For $t \in \mathbb{C}_{p}$ with $|t|_{p}<|q|_{p} p^{-\frac{1}{p-1}}$, put $f(x)=(1+q \xi t)^{\frac{x+y}{q}}$. By (1.1), we get

$$
\begin{aligned}
\int_{\mathbb{Z}_{p}}(1+q \xi t)^{\frac{x+y}{q}} d \mu_{0}(y) & =(1+q \xi t)^{\frac{x}{q}} \frac{\log (1+q \xi t)}{q\left((1+q \xi t)^{\frac{1}{q}}-1\right)} \\
& =\sum_{n=0}^{\infty} D_{n, \xi, q}(x) \frac{t^{n}}{n !}
\end{aligned}
$$

By (2.2) and (2.3), we obtain the following theorem.

Theorem 2.1 For $n \geq 0$, we have

$$
D_{n, \xi, q}(x)=\xi^{n} \int_{\mathbb{Z}_{p}}(x+y)_{n, q} d \mu_{0}(y)
$$

In (2.3), by replacing $t$ by $\frac{1}{\xi q}\left(e^{\xi t}-1\right)$, we have

$$
\sum_{n=0}^{\infty} D_{n, \xi, q}(x) \frac{1}{\xi^{n} q^{n}} \frac{\left(e^{\xi t}-1\right)^{n}}{n !}=e^{\frac{\xi t x}{q}} \frac{\frac{\xi t}{q}}{e^{\frac{\xi t}{q}}-1}=\sum_{n=0}^{\infty} B_{n}(x) \frac{\xi^{n}}{q^{n}} \frac{t^{n}}{n !}
$$

and

$$
\begin{aligned}
\sum_{n=0}^{\infty} \frac{D_{n, \xi, q}(x)}{\xi^{n} q^{n}} \frac{1}{n !}\left(e^{\xi t}-1\right)^{n} & =\sum_{n=0}^{\infty} \frac{D_{n, \xi, q}(x)}{\xi^{n} q^{n}} \sum_{m=n}^{\infty} \xi^{m} S_{2}(m, n) \frac{t^{m}}{m !} \\
& =\sum_{m=0}^{\infty} \sum_{n=0}^{m} \frac{D_{n, \xi, q}(x)}{\xi^{n} q^{n}} \xi^{m} S_{2}(m, n) \frac{t^{m}}{m !} .
\end{aligned}
$$

By (2.4) and (2.5), we obtain the following corollary.

Corollary 2.2 For $n \geq 0$, we have

$$
B_{n}(x)=\sum_{m=0}^{n} D_{m, \xi, q}(x) \xi^{-m} q^{n-m} S_{2}(n, m)
$$

By Theorem 2.1,

$$
\begin{aligned}
D_{n, \xi, q}(x) & =\xi^{n} \int_{\mathbb{Z}_{p}}(x+y)_{n, q} d \mu_{0}(y) \\
& =\xi^{n} q^{n} \sum_{l=0}^{n} \frac{1}{q^{l}} S_{1}(n, l) \int_{\mathbb{Z}_{p}}(x+y)^{l} d \mu_{0}(y) .
\end{aligned}
$$

By (1.2), we can derive easily that

$$
\begin{aligned}
\int_{\mathbb{Z}_{p}} e^{(x+y) t} d \mu_{0}(y) & =\frac{t}{e^{t}-1} e^{x t}=\sum_{n=0}^{\infty} B_{n}(x) \frac{t^{n}}{n !} \\
& =\sum_{l=0}^{\infty} \int_{\mathbb{Z}_{p}}(x+y)^{l} d \mu_{0}(y) \frac{t^{l}}{l !},
\end{aligned}
$$


and so

$$
B_{n}(x)=\int_{\mathbb{Z}_{p}}(x+y)^{n} d \mu_{0}(y) .
$$

By (1.6), (2.7), and (2.8), we obtain the following corollary.

Corollary 2.3 For $n \geq 0$, we have

$$
D_{n, \xi, q}(x)=\xi^{n} \sum_{l=0}^{n} q^{n-l} S_{1}(n, l) B_{l}(x)=\xi^{n} \sum_{l=0}^{n}\left|S_{1}(n, l)\right|(-q)^{n-l} B_{l}(x) .
$$

From now on, we consider twisted Daehee polynomials of order $k \in \mathbb{N}$ with q-parameter. Twisted Daehee polynomials of order $k \in \mathbb{N}$ with $q$-parameter are defined by the multivariant $p$-adic invariant integral on $\mathbb{Z}_{p}$ :

$$
D_{n, \xi, q}^{(k)}(x)=\xi^{n} \int_{\mathbb{Z}_{p}} \cdots \int_{\mathbb{Z}_{p}}\left(x_{1}+\cdots+x_{k}+x\right)_{n, q} d \mu_{0}\left(x_{1}\right) \cdots d \mu_{0}\left(x_{k}\right),
$$

where $n$ is a nonnegative integer and $k \in \mathbb{N}$. In the special case, $x=0, D_{n, \xi, q}^{(k)}=D_{n, \xi, q}^{(k)}(0)$ are called the Daehee numbers of order $k$ with q-parameter.

From (2.9), we can derive the generating function of $D_{n, \xi, q}^{(k)}(x)$ as follows:

$$
\begin{aligned}
& \sum_{n=0}^{\infty} D_{n, \xi, q}^{(k)}(x) \frac{t^{n}}{n !} \\
& \quad=\sum_{n=0}^{\infty} \xi^{n} q^{n} \int_{\mathbb{Z}_{p}} \cdots \int_{\mathbb{Z}_{p}}\left(\frac{x_{1}+\cdots+x_{k}+x}{q}\right) d \mu_{0}\left(x_{1}\right) \cdots d \mu_{0}\left(x_{k}\right) t^{n} \\
& \quad=\int_{\mathbb{Z}_{p}} \cdots \int_{\mathbb{Z}_{p}}(1+q \xi t)^{\frac{x_{1}+\cdots+x_{k}+x}{q}} d \mu_{0}\left(x_{1}\right) \cdots d \mu_{0}\left(x_{k}\right) \\
& =(1+q \xi t)^{\frac{x}{q}} \int_{\mathbb{Z}_{p}} \cdots \int_{\mathbb{Z}_{p}}(1+q \xi t)^{\frac{x_{1}+\cdots+x_{k}}{q}} d \mu_{0}\left(x_{1}\right) \cdots d \mu_{0}\left(x_{k}\right) \\
& =(1+q \xi t)^{\frac{x}{q}}\left(\frac{\log (1+q \xi t)}{q\left((1+q \xi t)^{\frac{1}{q}}-1\right)}\right)^{k} .
\end{aligned}
$$

Note that, by (2.9),

$$
D_{n, \xi, q}^{(k)}(x)=\xi^{n} q^{n} \sum_{m=0}^{n} \frac{S_{1}(n, m)}{q^{m}} \int_{\mathbb{Z}_{p}} \cdots \int_{\mathbb{Z}_{p}}\left(x_{1}+\cdots+x_{k}+x\right)^{m} d \mu_{0}\left(x_{1}\right) \cdots d \mu_{0}\left(x_{k}\right) .
$$

Since

$$
\int_{\mathbb{Z}_{p}} \cdots \int_{\mathbb{Z}_{p}} e^{\left(x_{1}+\cdots+x_{k}+x\right) t} d \mu_{0}\left(x_{1}\right) \cdots d \mu_{0}\left(x_{k}\right)=\left(\frac{t}{e^{t}-1}\right)^{k} e^{x t}=\sum_{n=0}^{\infty} B_{n}^{(k)}(x) \frac{t^{n}}{n !},
$$

we can derive easily

$$
B_{n}^{(k)}(x)=\int_{\mathbb{Z}_{p}} \cdots \int_{\mathbb{Z}_{p}}\left(x_{1}+\cdots+x_{k}+x\right)^{n} d \mu_{0}\left(x_{1}\right) \cdots d \mu_{0}\left(x_{k}\right) .
$$


Thus, by (2.11) and (2.12), we have

$$
\begin{aligned}
D_{n, \xi, q}^{(k)}(x) & =\xi^{n} q^{n} \sum_{m=0}^{n} \frac{S_{1}(n, m)}{q^{m}} B_{m}^{(k)}(x) \\
& =\xi^{n} \sum_{m=0}^{n} q^{n-m} S_{1}(n, m) B_{m}^{(k)}(x) \\
& =\xi^{n} \sum_{m=0}^{n}\left|S_{1}(n, m)\right|(-q)^{n-m} B_{m}^{(k)}(x) .
\end{aligned}
$$

In (2.10), by replacing $t$ by $\frac{1}{q \xi}\left(e^{\xi t}-1\right)$, we get

$$
\sum_{n=0}^{\infty} D_{n, \xi, q}^{(k)}(x) \frac{\left(e^{\xi t}-1\right)^{n}}{\xi^{n} q^{n} n !}=e^{\frac{\xi t x}{q}}\left(\frac{\frac{\xi t}{q}}{e^{\frac{\xi t}{q}}-1}\right)^{k}=\sum_{n=0}^{\infty} \frac{\xi^{n} B_{n}^{(k)}(x)}{q^{n}} \frac{t^{n}}{n !}
$$

and

$$
\begin{aligned}
\sum_{n=0}^{\infty} \frac{D_{n, \xi, q}^{(k)}(x)}{\xi^{n} q^{n}} \frac{1}{n !}\left(e^{\xi t}-1\right)^{n} & =\sum_{n=0}^{\infty} \frac{D_{n, \xi, q}^{(k)}(x)}{\xi^{n} q^{n}} \sum_{l=n}^{\infty} S_{2}(l, n) \xi^{l} \frac{t^{l}}{l !} \\
& =\sum_{m=0}^{\infty}\left(\xi^{m} \sum_{n=0}^{m} \frac{D_{n, \xi, q}^{(k)}(x)}{\xi^{n} q^{n}} S_{2}(m, n)\right) \frac{t^{m}}{m !}
\end{aligned}
$$

By (2.13), (2.14), and (2.15), we obtain the following theorem.

Theorem 2.4 For $n \geq 0$ and $k \in \mathbb{N}$, we have

$$
D_{n, \xi, q}^{(k)}(x)=\xi^{n} \sum_{m=0}^{n} q^{n-m} S_{1}(n, m) B_{m}^{(k)}(x)=\xi^{n} \sum_{m=0}^{n}\left|S_{1}(n, m)\right|(-q)^{n-m} B_{m}^{(k)}(x)
$$

and

$$
B_{n}^{(k)}(x)=\sum_{m=0}^{n} D_{m, \xi, q}^{(k)}(x) \xi^{-m} q^{n-m} S_{2}(n, m)
$$

Now, we consider the twisted Daehee polynomials of the second kind with q-parameter as follows:

$$
\hat{D}_{n, \xi, q}(x)=\xi^{n} \int_{\mathbb{Z}_{p}}(-y+x)_{n, q} d \mu_{0}(y) \quad(n \geq 0)
$$

In the special case $x=0, \hat{D}_{n, \xi, q}(0)=\hat{D}_{n, \xi, q}$ are called the twisted Daehee numbers of the second kind with q-parameter.

By (2.16), we have

$$
\hat{D}_{n, \xi, q}(x)=\xi^{n} q^{n} \int_{\mathbb{Z}_{p}}\left(\frac{-y+x}{q}\right)_{n} d \mu_{0}(y)
$$


and so we can derive the generating function of $\hat{D}_{n, \xi, q}(x)$ by (1.1) as follows:

$$
\begin{aligned}
\sum_{n=0}^{\infty} \hat{D}_{n, \xi, q}(x) \frac{t^{n}}{n !}= & \sum_{n=0}^{\infty} q^{n} \xi^{n} \int_{\mathbb{Z}_{p}}\left(\frac{-y+x}{q}\right)_{n} d \mu_{0}(y) \frac{t^{n}}{n !} \\
& =\sum_{n=0}^{\infty} q^{n} \xi^{n} \int_{\mathbb{Z}_{p}}\left(\begin{array}{c}
\frac{-y+x}{q} \\
n
\end{array}\right) d \mu_{0}(y) t^{n} \\
& =\int_{\mathbb{Z}_{p}}(1+q \xi t)^{\frac{-y+x}{q}} d \mu_{0}(y) \\
& =(1+q \xi t)^{\frac{x}{q}} \frac{\log (1+q \xi t)}{q\left((1+q \xi t)^{\frac{1}{q}}-1\right)}(1+q \xi t)^{\frac{1}{q}}
\end{aligned}
$$

From (1.3), (1.6), and (2.17), we get

$$
\begin{aligned}
\hat{D}_{n, \xi, q}(x) & =q^{n} \xi^{n} \int_{\mathbb{Z}_{p}}\left(\frac{-y+x}{q}\right)_{n} d \mu_{0}(y) \\
& =q^{n} \xi^{n} \int_{\mathbb{Z}_{p}} \sum_{l=0}^{n} \frac{S_{1}(n, l)}{q^{l}}(-y+x)^{l} d \mu_{0}(y) \\
& =\xi^{n} \sum_{l=0}^{n} S_{1}(n, l)(-1)^{l} \int_{\mathbb{Z}_{p}}(y-x)^{l} d \mu_{0}(y) q^{n-l} \\
& =\xi^{n} \sum_{l=0}^{n} S_{1}(n, l)(-1)^{l} B_{l}(-x) q^{n-l} \\
& =(-\xi)^{n} \sum_{l=0}^{n}\left|S_{1}(n, l)\right| B_{l}(-x) q^{n-l} .
\end{aligned}
$$

By (1.10), it is easy to show that $B_{n}(-x)=(-1)^{n} B_{n}(x+1)$. Thus, from (2.19), we have the following theorem.

Theorem 2.5 For $n \geq 0$, we have

$$
\hat{D}_{n, \xi, q}(x)=\xi^{n} \sum_{l=0}^{n} S_{1}(n, l)(-1)^{l} B_{l}(-x) q^{n-l}=\xi^{n} \sum_{l=0}^{n}\left|S_{1}(n, l)\right| B_{l}(x+1)(-q)^{n-l} .
$$

By replacing $t$ by $\frac{1}{q \xi}\left(e^{\xi t}-1\right)$ in $(2.18)$, we have

$$
\sum_{n=0}^{\infty} \hat{D}_{n, \xi, q}(x) \frac{1}{q^{n} \xi^{n}} \frac{\left(e^{\xi t}-1\right)^{n}}{n !}=e^{\frac{\xi t}{q}(x+1)} \frac{\frac{\xi t}{q}}{e^{\frac{\xi t}{q}}-1}=\sum_{n=0}^{\infty} \frac{\xi^{n} B_{n}(x+1)}{q^{n}} \frac{t^{n}}{n !}
$$

and

$$
\begin{aligned}
\sum_{n=0}^{\infty} \hat{D}_{n, \xi, q}(x) \frac{1}{q^{n} \xi^{n}} \frac{\left(e^{\xi t}-1\right)^{n}}{n !} & =\sum_{n=0}^{\infty} \frac{\hat{D}_{n, \xi, q}(x)}{q^{n} \xi^{n}} \sum_{m=n}^{\infty} S_{2}(m, n) \frac{(\xi t)^{m}}{m !} \\
& =\sum_{n=0}^{\infty}\left(\sum_{m=0}^{n} \hat{D}_{m, \xi, q}(x) S_{2}(n, m) q^{-m} \xi^{n-m}\right) \frac{t^{n}}{n !} .
\end{aligned}
$$

By (2.20) and (2.21), we obtain the following theorem. 
Theorem 2.6 For $n \geq 0$, we have

$$
B_{n}(x+1)=\sum_{m=0}^{n} q^{n-m} \xi^{-m} \hat{D}_{m, \xi, q}(x) S_{2}(n, m)
$$

Now, we consider higher-order twisted Daehee polynomials of the second kind with q-parameter. Higher-order twisted Daehee polynomials of the second kind with qparameter are defined by the multivariant $p$-adic invariant integral on $\mathbb{Z}_{p}$ :

$$
\hat{D}_{n, \xi, q}^{(k)}(x)=\xi^{n} \int_{\mathbb{Z}_{p}} \cdots \int_{\mathbb{Z}_{p}}\left(-x_{1}-\cdots-x_{k}+x\right)_{n, q} d \mu_{0}\left(x_{1}\right) \cdots d \mu_{0}\left(x_{k}\right),
$$

where $n$ is a nonnegative integer and $k \in \mathbb{N}$. In the special case, $x=0, \hat{D}_{n, \xi, q}^{(k)}=\hat{D}_{n, \xi, q}^{(k)}(0)$ are called the higher-order twisted Daehee numbers of the second kind with q-parameter.

From (2.22), we can derive the generating function of $\hat{D}_{n, \xi, q}^{(k)}(x)$ as follows:

$$
\begin{aligned}
& \sum_{n=0}^{\infty} \hat{D}_{n, \xi, q}^{(k)}(x) \frac{t^{n}}{n !} \\
& \quad=\sum_{n=0}^{\infty} \xi^{n} q^{n} \int_{\mathbb{Z}_{p}} \cdots \int_{\mathbb{Z}_{p}}\left(\frac{\frac{-x_{1}-\cdots-x_{k}+x}{q}}{n}\right) d \mu_{0}\left(x_{1}\right) \cdots d \mu_{0}\left(x_{k}\right) t^{n} \\
& \quad=\int_{\mathbb{Z}_{p}} \cdots \int_{\mathbb{Z}_{p}}(1+q \xi t)^{\frac{-x_{1}-\cdots-x_{k}+x}{q}} d \mu_{0}\left(x_{1}\right) \cdots d \mu_{0}\left(x_{k}\right) \\
& \quad=(1+q \xi t)^{\frac{x+k}{q}}\left(\frac{\log (1+q \xi t)}{q\left((1+q \xi t)^{\frac{1}{q}}-1\right)}\right)^{k} .
\end{aligned}
$$

By (2.22),

$$
\begin{aligned}
& \hat{D}_{n, \xi, q}^{(k)}(x) \\
& \quad=\xi^{n} q^{n} \sum_{m=0}^{n} \frac{S_{1}(n, m)}{q^{m}} \int_{\mathbb{Z}_{p}} \cdots \int_{\mathbb{Z}_{p}}\left(-x_{1}-\cdots-x_{k}+x\right)^{m} d \mu_{0}\left(x_{1}\right) \cdots d \mu_{0}\left(x_{k}\right) \\
& \quad=\xi^{n} q^{n} \sum_{m=0}^{n} \frac{S_{1}(n, m)}{(-q)^{m}} \int_{\mathbb{Z}_{p}} \cdots \int_{\mathbb{Z}_{p}}\left(x_{1}+\cdots+x_{k}-x\right)^{m} d \mu_{0}\left(x_{1}\right) \cdots d \mu_{0}\left(x_{k}\right) \\
& =\xi^{n} q^{n} \sum_{m=0}^{n} \frac{S_{1}(n, m)}{(-q)^{m}} B_{m}^{(k)}(-x) \\
& \quad=\xi^{n} \sum_{m=0}^{n} q^{n-m}\left|S_{1}(n, m)\right| B_{m}^{(k)}(-x) .
\end{aligned}
$$

From (1.10), we know that $B_{n}^{(k)}(-x)=(-1)^{n} B_{n}^{(k)}(k+x)$. Hence, by (2.24), we obtain the following theorem.

Theorem 2.7 For $n \geq 0$, we have

$$
\hat{D}_{n, \xi, q}^{(k)}(x)=\xi^{n} \sum_{m=0}^{n}(-1)^{m} q^{n-m} S_{1}(n, m) B_{m}^{(k)}(-x)=\xi^{n} \sum_{m=0}^{n}(-1)^{m} q^{n-m}\left|S_{1}(n, m)\right| B_{m}^{(k)}(x+k) .
$$


In (2.23), by replacing $t$ by $\frac{1}{q \xi}\left(e^{\xi t}-1\right)$, we get

$$
\sum_{n=0}^{\infty} \hat{D}_{n, \xi, q}^{(k)}(x) \frac{\left(e^{\xi t}-1\right)^{n}}{\xi^{n} q^{n} n !}=e^{\frac{\xi t}{q}(x+k)}\left(\frac{\frac{\xi t}{q}}{e^{\frac{\xi t}{q}}-1}\right)^{k}=\sum_{n=0}^{\infty} \frac{\xi^{n} B_{n}^{(k)}(x+k)}{q^{n}} \frac{t^{n}}{n !}
$$

and

$$
\begin{aligned}
\sum_{n=0}^{\infty} \frac{\hat{D}_{n, \xi, q}^{(k)}(x)}{\xi^{n} q^{n}} \frac{1}{n !}\left(e^{\xi t}-1\right)^{n} & =\sum_{n=0}^{\infty} \frac{\hat{D}_{n, \xi, q}^{(k)}(x)}{\xi^{n} q^{n}} \sum_{l=n}^{\infty} S_{2}(l, n) \xi l \frac{t^{l}}{l !} \\
& =\sum_{n=0}^{\infty}\left(\xi^{n} \sum_{m=0}^{n} \frac{\hat{D}_{m, \xi, q}^{(k)}(x)}{\xi^{m} q^{m}} S_{2}(n, m)\right) \frac{t^{n}}{n !} .
\end{aligned}
$$

By (2.25) and (2.26), we obtain the following theorem.

Theorem 2.8 For $n \geq 0$ and $k \in \mathbb{N}$, we have

$$
B_{n}^{(k)}(x+k)=\sum_{m=0}^{n} \hat{D}_{m, \xi, q}^{(k)}(x) \xi^{-m} q^{n-m} S_{2}(n, m)
$$

\section{Competing interests}

The author declares that they have no competing interests.

\section{Author's contributions}

The author contributed to the manuscript and typed, read, and approved the final manuscript.

\section{Acknowledgements}

The author is grateful for the valuable comments and suggestions of the referees. This paper was supported by the Sehan University Research Fund in 2014.

Received: 16 September 2014 Accepted: 17 November 2014 Published: 02 Dec 2014

\section{References}

1. Kim, T: On $q$-analogye of the $p$-adic log gamma functions and related integral. J. Number Theory 76(2), 320-329 (1999)

2. Kim, T: An invariant $p$-adic integral associated with Daehee numbers. Integral Transforms Spec. Funct. 13(1), 65-69 (2002)

3. Kim, T: q-Volkenborn integration. Russ. J. Math. Phys. 9(3), 288-299 (2002)

4. Comtet, L: Advanced Combinatorics. Reidel, Dordrecht (1974)

5. Kim, T, Kim, DS, Mansour, T, Rim, SH, Schork, M: Umbral calculus and Sheffer sequences of polynomials. J. Math. Phys. 52(8), 083504 (2013)

6. Roman, S: The Umbral Calculus. Dover, New York (2005)

7. Dolgy, DV, Kim, T, Lee, B, Lee, SH: Some new identities on the twisted Bernoulli and Euler polynomials. J. Comput. Anal. Appl. 15(3), 441-451 (2013)

8. Jeong, JH, Jin, JH, Park, JW, Rim, SH: On the twisted weak $q$-Euler numbers and polynomials with weight 0 . Proc Jangjeon Math. Soc. 16(2), 157-163 (2013)

9. Kim, DS, Kim, T: Daehee numbers and polynomials. Appl. Math. Sci. 7(120), 5969-5976 (2013)

10. Kim, YH, Hwang, KW: Symmetry of power sum and twisted Bernoulli polynomials. Adv. Stud. Contemp. Math. 18(2), 43-48 (2009)

11. Luo, QL: Some recursion formulae and relations for Bernoulli numbers and Euler numbers of higher order. Adv. Stud Contemp. Math. 10(1), 63-70 (2005)

12. Ozden, H, Cangul, IN, Simsek, Y: Remarks on q-Bernoulli numbers associated with Daehee numbers. Adv. Stud. Contemp. Math. 18(1), 41-48 (2009)

13. Simsek, Y: Generating functions of the twisted Bernoulli numbers and polynomials associated with their interpolation functions. Adv. Stud. Contemp. Math. 16(2), 251-278 (2008)

14. Simsek, Y: Generating functions for generalized Stirling type numbers, array type polynomials, Eulerian type polynomials and their applications. Fixed Point Theory Appl. 2013, 87 (2013)

15. Simsek, Y: On $p$-adic twisted $q$-L-function related to generalized twisted Bernoulli numbers. Russ. J. Math. Phys. 13(3), 340-348 (2006) 
16. Araci, S: Novel identities involving Genocchi numbers and polynomials arising from applications of umbral calculus. Appl. Math. Comput. 233, 599-607 (2014)

17. Araci, S, Acikgoz, M, Sen, E: On the von Staudt-Clausen's theorem associated with $q$-Genocchi numbers. Appl. Math. Comput. 247, 780-785 (2014)

18. Araci, S, Bagdasaryan, A, Özel, C, Srivastava, HM: New symmetric identities involving q-zeta type functions. Appl. Math. Inf. Sci. 8(6), 2803-2808 (2014)

19. Park, JW, Rim, SH, Kim, J: The twisted Daehee numbers and polynomials. Adv. Differ. Equ. 2014, 1 (2014)

20. Kim, DS, Kim, T, Kwon, HI, Seo, JJ: Daehee polynomials with q-parameter. Adv. Stud. Theor. Phys. 8(13), 561-569 (2014)

21. Kim, T, Lee, SH, Mansour, T, Seo, JJ: A note on $q$-Daehee polynomials and numbers. Adv. Stud. Contemp. Math. 24(2), 155-160 (2014)

10.1186/1687-1847-2014-304

Cite this article as: Park: On the twisted Daehee polynomials with q-parameter. Advances in Difference Equations 2014, 2014:304

\section{Submit your manuscript to a SpringerOpen ${ }^{\odot}$ journal and benefit from:}

- Convenient online submission

- Rigorous peer review

- Immediate publication on acceptance

- Open access: articles freely available online

- High visibility within the field

- Retaining the copyright to your article 